\title{
Beclin1 circulating levels and accelerated aging markers in COPD
}

\author{
Frédéric Schlemmer ${ }^{1,2}$, Laurent Boyer ${ }^{1,3}$, Thibaud Soumagne ${ }^{1}$, Audrey Ridoux ${ }^{1}$, Christos Chouaid ${ }^{1,4}$, Bernard Maitre ${ }^{1,2,4}$, \\ Sophie Lanone ${ }^{1}$, Serge Adnot ${ }^{1,3}$, Etienne Audureau ${ }^{5}$ and Jorge Boczkowski ${ }^{1,2}$
}

To the Editor:

Chronic obstructive pulmonary disease (COPD), so far simply defined by persistent airflow limitation mostly due to prolonged tobacco smoking exposure, is now clearly depicted as a heterogeneous and complex disease, with lung but also systemic manifestations such as sarcopenia, osteoporosis, or cardiovascular diseases ${ }^{1}$. Chronic exposure of lung cells to cigarette smoke can trigger the activation of several cellular processes such as oxidative stress, cellular senescence and autophagy. Induced in response to various stressful situations (e.g. starvation, hypoxia, DNA damage, or infection), autophagy is a major cellular adaptive pathway that, to a certain extent, helps maintain cellular homeostasis through phagolysosomal self-degradation of supernumerary or defective organelles and proteins ${ }^{2}$. Indeed, autophagy theoretically promotes cellular survival but can also favor cell death when this adaptive process is overwhelmed. The implication of autophagy in the pathogenesis of COPD initially looked controversial, with some data showing autophagy activation that favors apoptotic death of bronchial epithelial cells ${ }^{3}$, and some other demonstrating a defective autophagy that may promote cellular senescence ${ }^{4}$. However, recent studies uncovered the link between cigarette smoke-induced oxidative stress, autophagy-flux impairment, accumulation of autophagic vacuoles/aggresome-bodies, chronic inflammatory-apoptotic responses, premature senescence, and emphysema progression in the context of chronic cigarette smoke exposure or in COPD patient's lung tissue ${ }^{5-7}$. Although there are still areas of uncertainty, like specific steps and mechanisms by which

\footnotetext{
Correspondence/Letter: Frédéric. Schlemmer (frederic.schlemmer@aphp.fr) 'INSERM U955, Institut Mondor de Recherche Biomédicale and Université Paris Est-Créteil (UPEC), Faculté de Médecine, Créteil, France

${ }^{2}$ Unité de Pneumologie, APHP, Hôpital Henri Mondor, DHU-ATVB, Créteil,

France

Full list of author information is available at the end of the article
}

autophagy is impaired, strategies aiming to counteract these phenomena have already emerged as a way to prevent or limit the consequences of chronic cigarette-smoke exposure.

Molecular pathways triggering autophagy are complex and may involve the class III Bcl-2 interacting protein (Beclin1)/phosphoinositol-3-kinase complex, which then participates in the induction and the initial steps of autophagy $^{2}$. A recent study showed that healthy centenarians have increased circulating Beclin1 protein levels in comparison to a population of young healthy subjects or patients with myocardial infarction ${ }^{9}$. Thus, as it has been shown that the induction of autophagy may promote longevity, the authors have suggested a relationship between the increased level of this potential biomarker of autophagy and the exceptional longevity of these patients.

Given that cellular senescence is involved in COPD pathophysiology and that autophagy defect may be a trigger of this process, we hypothesized that circulating Beclin1 levels, taken as a reflect of autophagy process efficiency, are reduced in COPD patients. We therefore tested whether circulating Beclin1 levels are reduced in COPD patients and if so, if this reduction is linked to telomere shortening, a hallmark of senescence. Finally, as deficient autophagy may also be implicated in many pathophysiological processes such as cardiovascular diseases, neuromuscular disorders, or bone loss, we also tested whether Beclin1 level is linked to systemic manifestations of COPD. For that, we took advantage of a cohort of 301 participants recruited at the Henri Mondor Teaching Hospital, Creteil France (COPD, $n=100$; smokers $n=100$ and non-smoker patients $n=101$ ), already thoroughly phenotyped with a special focus on aging-related markers such as arterial stiffness (aortic pulse-wave velocity, PWV), bone mineral density (BMD), 
appendicular skeletal muscle mass (ASMM), pinch and grip strengths, insulin resistance, renal function, and telomere length in circulating leucocytes ${ }^{10}$. In 280 (COPD $n=92$, smokers $n=93$, non-smokers $n=95)$ of the 301 patients for whom a serum sample was available, we performed a quantitative evaluation of circulating Beclin1 protein level (ELISA Kit for Beclin1; cat. no. E98557Hu, Uscn Life Science Inc., Wuhan, China). Descriptive results are given as numbers and percentages for categorical data, and means ( \pm standard deviation) for continuous variables. Unadjusted comparisons of Beclin1 levels between study groups were conducted using one-way ANOVA for overall significance, and post hoc $t$-tests for pairwise comparisons applying Sidak correction for test multiplicity. Linear regression was used to compare Beclin1 levels across the three groups while adjusting for age. Associations between Beclin1 and biological parameters were assessed by computing Pearson correlation coefficients. All analyses were performed using Stata 14.1 (StataCorp, USA).

\section{Results}

Clinical characteristics (except pulmonary function tests) did not differ across the three groups, but COPD patients had a higher pack-year value compared to control smokers. A statistically significant negative trend was found in serum Beclin1 protein level of non-smokers, smokers, and COPD patients $(p=0.022$; Fig. 1$)$. Beclin1 protein level was correlated to age, degree of airway obstruction, telomere length, appendicular skeletal muscle mass index, and grip strength (Table 1). None of the 27 chemokines and growth factors tested was correlated to Beclin1 level. After adjustment for age, the statistically significant negative trend between serum Beclin1 protein level in each group persisted (non-smokers: $2.31 \pm 0.23$, smokers: $1.66 \pm 0.23$, and COPD: $1.52 \pm 0.24 \mathrm{ng} / \mathrm{mL}$, respectively; $p=0.036$ ). In COPD patients, Beclin1 protein level was correlated to pulse-oxygen saturation $(R=$ $0.25 ; p=0.024)$, telomere length $(R=0.26 ; p=0.014)$, and TNF- $\alpha$ levels $(R=0.35 ; p=0.001)$.

\section{Discussion}

We show a significant decrease in serum Beclin1 protein level, a key regulator of autophagy, in smokers and even more in COPD patients compared to healthy controls. Autophagy induction and apoptosis have been widely described in bronchial and alveolar epithelial cells exposed to cigarette smoke extracts in vitro and in lung tissues of COPD patients ${ }^{3}$. However, evidence on the implication of Beclin1 in the cellular consequences of cigarette smoke exposure are limited to its capacity to permit autophagic-dependant apoptosis induced by acute cigarette smoke exposure ${ }^{11}$. Our results suggest another potential role of Beclin1 in the regulation of

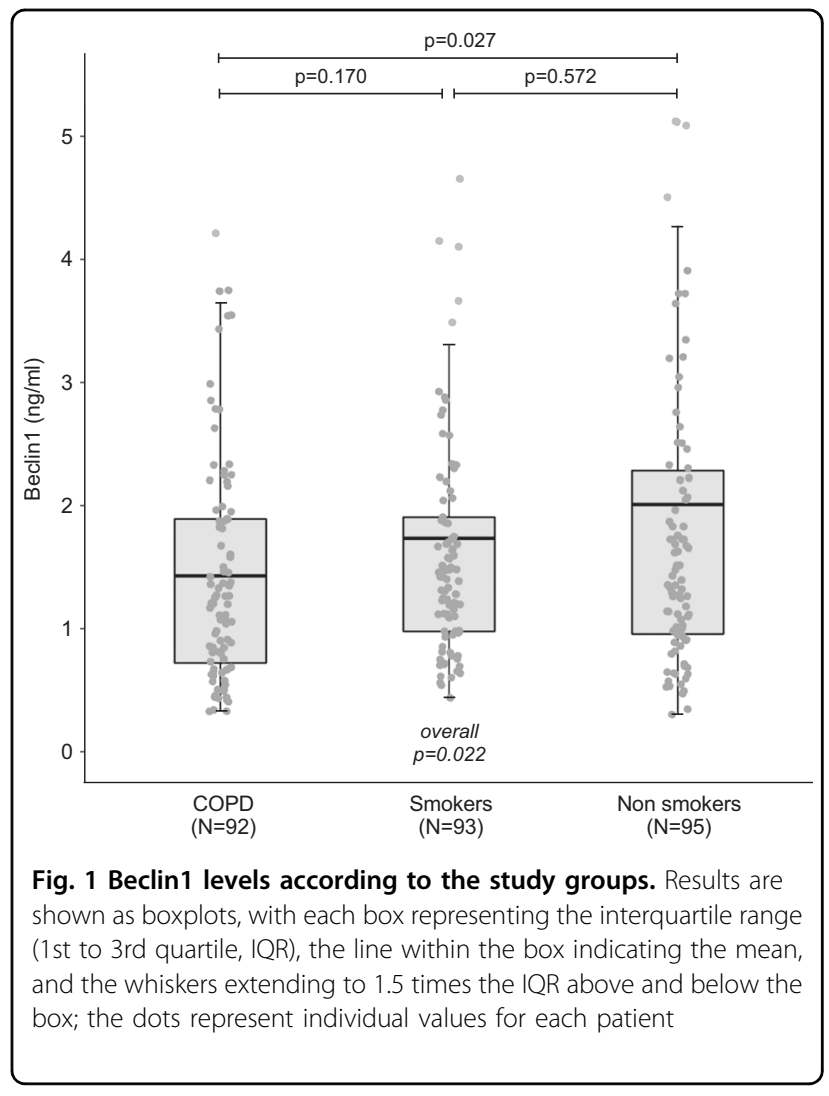

autophagy in the specific context of chronic cigarettesmoke exposure. Indeed, accumulation of autophagic vacuoles/agresomes observed in the lungs of patients with severe $\mathrm{COPD}^{3}$ already reflects an acquired defect in autophagic process during COPD, attributed to an autophagy-flux impairment that may be accessible to corrective therapies to prevent cellular senescence and emphysema ${ }^{8}$. Thus, Beclin1 circulating levels decline might also participate to autophagy impairment during COPD.

Furthermore, we show a correlation between the level of circulating Beclin1 and the degree of airflow obstruction, which is consistent with a progressive defect in autophagy during COPD, and also with two features of accelerated aging already associated to COPD, cellular senescence $^{12}$ and muscle wasting ${ }^{10}$, respectively evaluated by telomere length, ASMM and grip strength. Of course, the exact relationship between autophagy and cellular senescence remains unclear ${ }^{13}$, especially in the specific context of COPD, and the real implication of autophagy in skeletal muscle dysfunction is still deba$\operatorname{ted}^{14}$. Beclin1 has a pivotal role in the regulation of cell survival, apoptosis, and autophagy, especially via its interaction with anti-apoptotic proteins of the Bcl-2 family (Bcl-2, Bcl-XL, Bcl-w, and Mcl-1), the BH3 domain of Beclin1 important for the binding to the $\mathrm{BH} 3$ 
Table 1 Main subjects characteristics and Pearson correlation coefficients with Beclin1 level

\begin{tabular}{|c|c|c|c|c|c|}
\hline & & $N$ & Mean ( \pm SD) & Correlation coefficient with Beclin1 level & $p$-value* \\
\hline Age, years & & 279 & $59.02( \pm 7.76)$ & -0.13 & 0.027 \\
\hline $\mathrm{BMl}, \mathrm{kg} / \mathrm{m}^{2}$ & & 279 & $26.03( \pm 4.69)$ & 0.08 & 0.182 \\
\hline Pack-years & & 276 & $27.61( \pm 27.62)$ & -0.10 & 0.088 \\
\hline \multicolumn{6}{|l|}{ Pulmonary function parameters } \\
\hline FEV1, \% predicted & & 279 & $86.56( \pm 29.20)$ & 0.12 & 0.039 \\
\hline FVC, \% predicted & & 278 & $93.59( \pm 21.49)$ & 0.07 & 0.263 \\
\hline $\mathrm{DL}_{\mathrm{co}}, \%$ predicted & & 227 & $76.12( \pm 19.19)$ & -0.01 & 0.906 \\
\hline $\mathrm{PaO}_{2}, \mathrm{mmHg}$ & & 91 & $77.47( \pm 10.32)$ & 0.18 & 0.086 \\
\hline $\mathrm{SpO}_{2}, \%$ & & 239 & $96.59( \pm 1.35)$ & 0.09 & 0.178 \\
\hline 6-min walking distance, $\mathrm{m}$ & & 239 & $556.37( \pm 100.56)$ & 0.05 & 0.475 \\
\hline \multicolumn{6}{|l|}{ Systemic manifestations } \\
\hline Pulse wave velocity, $\mathrm{m} / \mathrm{s}$ & & 271 & $11.55( \pm 2.32)$ & -0.04 & 0.488 \\
\hline ASMMI, $\mathrm{kg} / \mathrm{m}^{2}$ & & 270 & $7.49( \pm 1.34)$ & 0.21 & 0.001 \\
\hline BMD, total lumbar, $\mathrm{g} / \mathrm{cm}^{2}$ & & 275 & $1.11( \pm 0.18)$ & 0.03 & 0.619 \\
\hline BMD, hips, g/cm² & & 275 & $0.98( \pm 0.14)$ & 0.10 & 0.108 \\
\hline Grip test, kg & & 244 & $37.47( \pm 12.26)$ & 0.13 & 0.046 \\
\hline Glomerular flow rate, $\mathrm{mL} / \mathrm{min}$ & & 261 & $93.34( \pm 48.78)$ & 0.02 & 0.802 \\
\hline HOMA-IR & & 265 & $2.65( \pm 2.81)$ & 0.05 & 0.465 \\
\hline \multicolumn{6}{|l|}{ Biological parameters } \\
\hline Telomere length (T/S) ratio & & 274 & $0.42( \pm 0.11)$ & 0.17 & 0.005 \\
\hline $\mathrm{IL}-6, \mathrm{pg} / \mathrm{mL}$ & & 271 & $18.07( \pm 15.97)$ & -0.01 & 0.905 \\
\hline IL-8, pg/mL & & 271 & $46.94( \pm 9.88)$ & -0.09 & 0.155 \\
\hline TNF-a, pg/mL & & 271 & $89.52( \pm 134.53)$ & 0.07 & 0.228 \\
\hline \multirow[t]{2}{*}{ MCP-1, pg/mL } & & 271 & $41.66( \pm 22.44)$ & -0.02 & 0.800 \\
\hline & & N & $N(\%)$ & Mean $( \pm S D)$ & $p$-value \\
\hline \multirow[t]{2}{*}{ Gender } & Men & 279 & 185 (66.31\%) & $1.80( \pm 1.45)$ & 0.230 \\
\hline & Women & & $94(33.69 \%)$ & $1.58( \pm 1.40)$ & \\
\hline \multirow[t]{2}{*}{ FEV1, \% predicted } & $\geq 50$ & 279 & 241 (86.38\%) & $1.80( \pm 1.50)$ & 0.035 \\
\hline & $<50$ & & $38(13.62 \%)$ & $1.27( \pm 0.73)$ & \\
\hline
\end{tabular}

${ }^{*} p$-values for Pearson correlation coefficients; bolded results are statistically significant at the $p<0.05$ level

$B M I$ body mass index, FEV1 forced expiratory volume in $1 \mathrm{~s}, \%$ predicted percentage of the predicted value, $F V C$ forced vital capacity, SpO $\mathrm{O}_{2}$ oxygen saturation by pulse oximetry, $A S M M I$ appendicular skeletal muscle mass index, BMD bone mineral density, HOMA-IR homeostatic model assessment of insulin resistance, $I L$ interleukin, TNF- $a$ tumor necrosis factor alpha, MCP-1 monocyte chemotactic protein-1, SD standard deviation

domain of the pro-apoptotic factors Bak, Bad, and Bim to Bcl-XL, and the activation of the Beclin1-interacting complex that generates phosphatidylinositol-3-phosphate, which promotes autophagosomal membrane nucleation and is indispensable for autophagy ${ }^{15}$. It now seems necessary to further investigate its potential regulatory role in the process of accelerated aging associated to chronic cigarette smoke exposure and COPD, at lung but also at systemic level.
In conclusion, although our results need to be confirmed in other cohorts of COPD patients or with other potential biomarkers of autophagy, they support the hypothesis of a relationship between autophagy deficiency and COPD pathogenesis. They also incite to refine our knowledge on the complex mechanisms linking defective autophagy and cellular senescence in the progressive pathogenesis of COPD and of its systemic manifestations, with a special focus on Beclin1. 


\section{Acknowledgements}

We gratefully acknowledge Lucie Bizard for helping to perform the ELISA, Elisabeth Marcos for the management of telomere length measurements, and Bruno Housset for his supervision.

\section{Author details}

'INSERM U955, Institut Mondor de Recherche Biomédicale and Université Paris Est-Créteil (UPEC), Faculté de Médecine, Créteil, France. ${ }^{2}$ Unité de Pneumologie, APHP, Hôpital Henri Mondor, DHU-ATVB, Créteil, France. ${ }^{3}$ Département de Physiologie-Explorations Fonctionnelles, APHP, Hôpital Henri Mondor, DHU-ATVB, Créteil, France. ${ }^{4}$ Département de Pneumologie et Pathologie Professionnelle, Centre Hospitalier Intercommunal, DHU-ATVB, Créteil, France. ${ }^{5}$ Département de Santé Publique, Unité de Recherche Clinique (URC-Mondor), APHP, Hôpital Henri Mondor, IMRB EA7376, Clinical Epidemiology and Aging (CEpiA), Créteil, France

\section{Conflict of interest}

The authors declare that they have no conflict of interest.

\section{Publisher's note}

Springer Nature remains neutral with regard to jurisdictional claims in published maps and institutional affiliations.

\section{Received: 21 July 2017 Revised: 21 November 2017 Accepted: 22} November 2017

Published online: 05 February 2018

\section{References}

1. Barnes, P. J. et al. Systemic manifestations and comorbidities of COPD. Eur. Respir. J. 33, 1165-1185 (2009).

2. Choi, A. M. K. et al. Autophagy in human health and disease. N. Engl. J. Med. 368, 1845-1846 (2013).
3. Chen, Z.-H. et al. Egr-1 regulates autophagy in cigarette smokeinduced chronic obstructive pulmonary disease. PLOS ONE 3, e3316 (2008).

4. Fujii, S. et al. Insufficient autophagy promotes bronchial epithelial cell senescence in chronic obstructive pulmonary disease. Oncoimmunology 1, 630-641 (2012).

5. Bodas, M. et al. Lactosylceramide-accumulation in lipid-rafts mediate aberrantautophagy, inflammation and apoptosis in cigarette smoke induced emphysema. Apoptosis 20, 725-739 (2015).

6. Tran, I. et al. Role of cigarette smoke-induced aggresome formation in chronic obstructive pulmonary disease-emphysema pathogenesis. Am. J. Respir. Cell Mol. Biol. 53, 159-173 (2015).

7. Bodas, M. et al. Master autophagy regulator transcription factor EB regulates cigarette smoke-induced autophagy impairment and chronic obstructive pulmonary disease-emphysema pathogenesis. Antioxid. Redox Signal 27, 150-167 (2017).

8. Bodas, M. et al. Augmenting autophagy for prognosis based intervention of COPD-pathophysiology. Respir. Res 18, 83 (2017).

9. Emanuele, E. et al. Can enhanced autophagy be associated with human longevity? Serum levels of the autophagy biomarker beclin-1 are increased in healthy centenarians. Rejuvenation Res 17, 518-524 (2014).

10. Boyer, L. et al. Aging-related systemic manifestations in COPD patients and cigarette smokers. PLOS ONE 10, e0121539 (2015).

11. Kim, H. P. et al. Autophagic proteins regulate cigarette smoke-induced apoptosis: protective role of heme oxygenase-1. Autophagy 4, 887-895 (2008).

12. Savale, L. et al. Shortened telomeres in circulating leukocytes of patients with chronic obstructive pulmonary disease. Am. J. Respir. Crit. Care Med 179 566-571 (2009).

13. Gewirtz, D. A. Autophagy and senescence: a partnership in search of definition. Autophagy 9, 808-812 (2013).

14. Hussain, S. N. A. et al. Role of autophagy in COPD skeletal muscle dysfunction. J. Appl. Physiol. 114, 1273-1281 (2013).

15. Levine, B. et al. Bcl-2 family members: dual regulators of apoptosis and autophagy. Autophagy 4, 600-606 (2008). 